\title{
Computer-Mediated Communication Portal Implementation Framework: A Higher Education Institutional Perspective
}

\author{
https://doi.org/10.3991/ijet.v15i03.11641 \\ Emmanuel Dortey Tetteh ${ }^{(凶)}$, Zhiguang Qin \\ University of Electronic Science and Technology of China, Chengdu, China \\ edtetteh@gmail.com \\ Benjamin Kwofie \\ Koforidua Technical University, Koforidua, Ghana
}

\begin{abstract}
Computer-mediated communication (CMC) portal services provision through information technologies (IT) in higher educational institutions (HEIs) should not be an impossible task considering the growth in information systems (IS) and an upsurge of internet users. There have been numerous efforts aimed at implementing CMC portals by HEIs but just a few could be said to be successful. This research aims to develop a framework that can help to provide a better understanding of how to manage the entire implementation process to bring the expected advantages to institutions implementing it. Results of past research had been studied to develop the conceptual framework utilizing significant theories in the field of information system implementation and institutional change. The framework addresses the adoption, implementation as well as institutionalization stages of CMC portal implementation, and several institutional contextual factors influencing implementation efforts within the stages.
\end{abstract}

Keywords-Information systems, CMC portal, adoption, implementation, institutionalization

\section{Introduction}

This research looks at the implementation of an information system (computermediated communication (CMC) portal) which serves as an aid for the delivering and supporting of teaching, learning, and administration of students within higher educational institutions (HEIs). Computer-mediated communication (CMC) within the institutional context can be defined as any communication patterns between students, lecturers, and administrators mediated through the utilization of the computer system. CMC over the years has become an integral component of communication within institutions around the world after its existence from the creation of the ARPANET produced unexpected benefits regarding email from the 1970s. There have been many research publications on the implementation of CMC within institutions and organizations and table 1 outlines some of these within the past years. 
There has been much research conducted on the implementation of CMC within institutions and organizations within the past years. Some of them include the examination of the uses and effects of a computer-based message system within an organizational setting which brought out the findings that no significant relationship between the amounts of time a respondent reported using a computer system and their perceptions of how appropriate computers are for communication tasks. However, heavy users found TFM substitutable for face-to-face communication[1], the examination of the behavior and attitudinal changes of "online" communication, using actual messages sent via IRC which indicated that computer-mediated communication led to the behavior disinhibition due to the lack of regulating cues[2], the examination of the recent developments in CMC research for educational environments which brought out the findings that Partial advantages of CMC in writing, task-focused discussion, collaborative decision-making, group work, and active involvement in knowledge construction during group interactions[3], the examination of the effect of text-based synchronous computer-mediated communication (SCMC) on second language acquisition which also indicated Text-based SCMC could make a larger difference on SLA than other ways of communication. Also, the learners may benefit more from SCMC tasks if they are grouped into pairs or small groups and participate in SCMC interactions weekly [4].

\section{CMC in Higher Education Institutions}

Even though studies by researchers and HEIs into the nature and utilization of information systems, specifically $\mathrm{CMC}$, has been done and still ongoing, the understanding of the whole institutional approach to the entire implementation process (adoption, implementation, and institutionalization) by HEIs is still on the drawback. Institutions or organizations incur huge investment costs in the adoption and implementation of information systems innovations but they may not achieve the expected results and outcomes because the adopted information system is not effectively utilized [5] [6].

Considering the $\mathrm{CMC}$ portal implementation advantages just as the high failure rate seen in practice, this research aim is to develop a framework that can help to provide a better understanding of how to manage the entire process to bring the expected advantages to institutions that have implemented or planning to.

\subsection{Systems for computer-mediated communication}

Various systems can be utilized for computer-mediated communication portal in education. For asynchronous communication, a simple email list allows a group of students to get in touch with one another. On the other hand, the internet supports a wide scope of web forums, newsgroups as well as Web Base Education (WbE) application that aims at delivering online technological classes, offering a flexible use of means and tools[7]. For synchronous communication, there are text-based chat tools, instant messaging, and audio- and video-conferencing systems. Computer conferencing systems are more specialist tools designed to support group communication, primarily via asyn- 
chronous discussion, but also encompassing synchronous communication. Various institutions around the world have developed their online portals, which can incorporate the transmission of teaching materials with facilities for collaborative work as well as asynchronous and synchronous discussions. In recent years Virtual Learning Environments (VLEs) such as Blackboard, Moodle and WebCT have become widely available, and many universities are using these systems with their students [8].

VLEs typically include asynchronous discussion forums and synchronous chat tools (as well as other facilities for online course delivery and assessment). They may also provide further communication tools such as wikis and blogs which can be transmitted via smart mobile devices which are used by most people in both developed and developing countries as "There is no gender and age difference regarding the purpose of using smart mobile devices"[9].

The CMC Portal system introduction in HEIs for the support of teaching, learning, and administration of students can be said to be an information systems (IS) or an information technology (IT) innovation that brings onboard new ways for the conveyance of education.

\section{Framework Structure}

\subsection{Framework development}

The research approach utilized for the design of the proposed CMC portal implementation framework is based on the IS/IT implementation process framework [10]. The implementation of an IS like the CMC portal within any HEI setting is not a straight forward process but rather incorporate sub-processes that come together to achieve a successful and effective implementation. The deployment of information systems in institutions have made use of the development of IS implementation and Innovation implementation frameworks. Some of these frameworks utilized by past researchers in their works include the implementation of Material requirements planning (MRP) system [10], the Integrated Service Digital Network (ISDN) implementation [11], the Electronic Data Interchange (EDI) implementation [12] and the Object-Oriented Programming Languages (OOPL) assimilation [13].

A useful framework proposed that integrates the elements of change [14] is the information systems implementation framework [15]. The framework proposes that for any institutions or organizations to implement a new IS, it must go through six sequential (6) stages of a process which include initiation, adoption, adaptation, acceptance, use, and incorporation.

There was a further modification of the framework later which replaced the last two stages consisting of use and incorporation with routinization and infusion that recommend that institutions move from unfreezing to refreezing stages within the implementation process [10]. They went on to contend that, some of the stages can happen in parallel when classified as activities although [15] proposed a sequential stages order, and that the framework can include numerous IT applications and implementation processes within institutions. This framework [10] does a great deal of help for institutions 
that wants to implement an IS innovation by highlighting the significance of a full understanding of the entire IS implementation process from its introduction until its implantation. But institutions must make sure that the implemented IS innovation's functionality fit with the institutions' procedures and processes because organizations cannot just expect the adoption of IS alone to generate the benefit of superior services and reduced operational costs [16] [17].

The proposed CMC portal implementation framework in this research is an extension of the IS/IT implementation process framework [10] that suggested that such a technological innovation as a CMC portal goes through a process of an origination of an idea to its physical deployment as well as sub-processes or phases that occur in series which outlines directly or indirectly activities that the institution undertakes with the aim of having an understanding of the purpose of the system.

Figure 1 is the IS/IT framework [10] consisting of elements of change [14] outlining the different stages of implementation. "Infusion is the final stage of a six stages sequential model of IT implementation. In the first stage, initiation is the process of scanning organizational problems and opportunities and of undertaking IT solutions and is associated with [14] unfreezing stage. The next two stages, adoption and adaptation, represent Lewin's change stage. Adoption implies to get organizational supporting the IT implementation while adaptation encompasses its development, installation, and maintenance. The last three stages, acceptance, routinization, and infusion can be mapped into Lewin's refreezing stage. The acceptance stage is the effort undertaken to induce organizational members to commit to using the technology. Routinization represents the alterations that occur within the work system to account for the IT such that it is no longer perceived as new. Finally, infusion occurs as the IT becomes more deeply embedded within the organization's groups', or individuals' work systems" [18]. The five contextual factors which impact institutional IS implementation efforts [15] are also presented below.

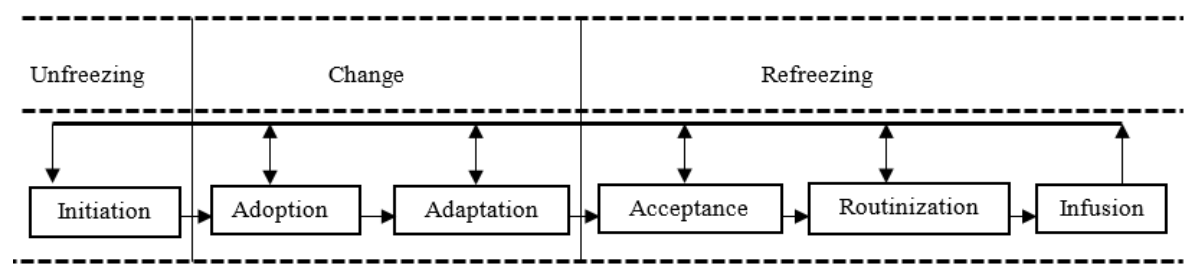

Fig. 1. IS implementation framework [10]

\subsection{Contextual factors influencing IS efforts in institutions}

In the introduction of any technological innovations, five vital factors (individual, structural, technological, task and environmental) [15], do add to it being successful or non-successful in any institution. A brief discussion is presented below concerning these five factors.

Individual: The literature on the individual factors as indicated [19] by researchers in the field of innovation mostly looked out for the adoption behaviors and that four of 
these individual variables include job tenure, cosmopolitan, educational background, and institutional role involvement. There was a great relationship seen between an individual's attitude towards change and these four variables and that these individual's attitudes towards change appear during undertakings involving innovations.

In as much as positive relationships are frequently seen between the job tenure variable and the individual in innovation research, there was a negative association between job tenure variable and utilization as well as satisfaction but for performance, there was mixed finding concerning an individual's job tenure [19].

With the Cosmopolitanism variable, there has been the contention that through the search for a broader perspective from people outside the institution by individuals, positive associations can happen. Regardless of the way that confirmation of this exists with positive relationships being identified between adoption and that of professionalism likewise incorporation, negative associations have been found with an individual's adoption of such innovations.

With the education variable, there exists a relation between an individual's attitude towards change and that of education and that, the more probable the receptivity of the innovation if the individual has a higher education level. Even though positive results have consistently been found in institutional innovation studies in the IS literature, there were negative associations seen with utilization as well as satisfaction and a mixed one when it comes to performance [19].

With institutional role involvement, there can be a positive association towards the adoption of an innovation, when an individual is involved in a managerial role. There has also been an association between top management and user participation in an institutional innovation process and that of an individual's attitude towards change [19].

Structural: The structural contextual factor deals with both formal as well as informal structural arrangements that influence technological innovations within institutions. Literature by Kwon and Zmud outlined three variables (specialization, centralization, and formalization) in connection to behaviors exhibited during any innovation's initiation and adoption stages.

With specialization, it has been contended that it is fundamental and has positive outcomes because of technical rationality but be as it may, there is the potential for it to increase social and political conflict within institutional innovations. There have been some negative outcomes concerning adoption although positive relations exist with initiation, adoption, and performance.

For centralization, there can be a negative effect when a bounded point of view exists alongside decreased autonomy whereas positive effect can be seen in the form of increased efficiency. There have been positive associations seen concerning adoption and utilization as well as negative associations concerning initiation, adoption, adaptation, and performance in a couple of cases.

On account of formalization, several findings have come out where there is the potential of functional differentiation levels to develop clear work definition and process but lead to less autonomy at the same time concerning initiation in studies involving innovation. With adoption, adaptation, utilization as well as performance, Positive associations have been seen. 
Technological: Technological characteristics that tend to affect most institutional innovation behaviors which have been studied by most researchers include compatibility, complexity, and relative advantage.

Compatibility becomes essential in the sense that, for any successful institutional innovation, there must be a fit between that technological innovation and the institution and that fit is seen to be very necessary for the attitude of an individual towards change, how convenience the change is to the individual, as well as the change in power for that individual. There have been positive associations concerning the adoption and adaptation stages during implementation.

Complexity in the technological factor refers to the level of difficulty stakeholders encounter in the understanding and utilization of the innovation. In the occurrence where there isn't the need for greater growth as well as achievement in the institution, this variable has been seen to affect users negatively. Institutional innovations have been opposed because of the lack of skills and understanding from users and for this resistance not to happen which will aid the successful implementation, there must be a greater perception among stakeholders on the simplicity of utilizing and understanding that innovation. Negative just as the positive relationship was highlighted in his work [19].

With relative advantage variable, any institutional innovations must give more prominent advantages as compared to the current or previous ones and when this is not achieved, the utilization of the innovation will be negatively affected which will be a result of the stakeholders seeing it be less useful. Despite the negative associations of this variable seen concerning innovation in most studies, reported generally positive relations in the adoption and adaptation stages in implementation.

Task: The two means by which task can be looked at, are task uncertainty and Hackman \& Oldham's five-key task-related attributes (task autonomy, responsibility, variety, identity, and feedback).

With task uncertainty, institutional conduct is extraordinarily affected by it and for the achievement of any institutional task, it plays the role of a multi-faceted construct that reveals the level of routinization, exceptions, and programmability. The positive impact is accomplished by this variable where the task difficulty inspires initiation and use practices, likewise and a negative impact where it acts as an obstacle to implementation [19].

The focal point of interest of autonomy is how the individual exercises personal control over the task assigned to them and that stakeholders' motivation, satisfaction, generation of idea and performance can be increased by a greater level of autonomy.

The responsibility variable can influence institutional innovation. This is so because, the level of authority placed on an individual in seeing to the completion or improvement in the behavior of a given task, has a relation to the institutional innovation implementation. They also revealed through their work that there have been positive associations of this variable concerning satisfaction and performance.

With the variety variable, it is believed that when the task is routinized and simplified, and where the tasks performer does require some 'value addition', higher performance and satisfaction will not likely be achieved. With the perception that routinizing 
tasks may disconnect stakeholders from the change as well as bring about more resistance to the change, there have still been positive associations seen concerning adoption, adaptation, utilization, satisfaction, and that of performance.

The conscious mind reasoning about an assigned task by an individual refers to the Identity variable. The potential for an individual to increase their involvement in the task that ultimately will bring about more innovative behaviors will happen when that individual relates to and believes in that assigned task. A positive relationship has been seen concerning satisfaction but with performance, inconclusive relations were seen.

Lastly, the feedback variable of the task factor is when the task performance levels of an individual are made known to that person through a mechanism. There is the belief according to theories of learning and reinforcements that, the frequency of feedback and the level of innovation displayed in behavior can bring about a positive relation. A positive relationship has been seen generally concerning satisfaction but with performance, inconclusive relations were seen [19].

Environmental: The environmental factors can be seen in two ways; seeing the environment as being a source of information and as being a stock of resources. Heterogeneity and uncertainty are the variables when the environment is seen as a source of information and competition and resource concentration/dispersion are the variable when the environment is seen as a stock of resources.

On account of heterogeneity, environmental entities similarities by which the institution is required to interact with are useful for innovation within the institution. Positive relations were seen in some of the studies. The unforeseen events from the environment are believed to inspire, as opposed to holding back innovation as various institutional roles openings come about due to the multiplicity created in the environment [19].

With the uncertainty variable, circumstances of both unsteadiness and confusion, as well as steadiness for an institution, can come about because of the variability in the environment. There is the perception out there that, an institution is invigorated to make every effort for survival and growth rather than essentially give in where there exists uncertainty. There has been an identification of positive associations with this variable [19], and negative associations are believed to exist concerning adoption due to the uncertainty variable posing as a constraint on the degree and measure of innovation available to institutions who want to implement.

The environment's capacity concerning scarcity of resources along with population density is the issue of concern with the competition variable. There is the belief that the likelihood of innovative activities increases with increases in competition. There is a positive association between adoption and competition stages [19].

On the account of the concentration/dispersion variable, there is the conviction that there can be a facilitation of institutional learning which can also result in innovation in the institutional effort to vie for the inadequate resources when there is a concentration of resources at a particular location in an environment. Positive relations have been established between adoption and incorporation [19]. 


\subsection{Proposed CMC portal implementation framework}

The proposed institutional CMC portal implementation framework which is displayed in figure 2 depicts a complex connection of activities and contextual factors cooperating to accomplish an expected institutional outcome and furthermore connotes a strategic approach in implementation which is based on the IS implementation process framework, organizational innovation process, innovation diffusion theory and planned change process.

The framework encompasses a straight succession of procedures and activities that highlight a logical movement of an institution's endeavors to introduce an innovation such as the CMC portal for online/distance and on-campus learning purposes. This is in no way, a recommendation that it is a standard as a general rule as captured by the two-way arrow in the diagram below and as highlighted by both cases in the research but instead, this framework aims at highlighting the vital stages in an institutional information system (CMC portal) implementation process, alongside the contextual factors that shape its outcomes. From the diagram below, there are three (3) broad phases of the educational change process (Adoption, implementation, and institutionalization) frequently found in the institutional innovation and IS innovation kinds of literature and seven (7) sub-categories which include initiation, promotion, adoption, adaptation, acceptance, routinization, and infusion. Adoption, implementation, and institutionalization are useful concepts for organizing our thoughts around educational change. Adoption is deciding to push a specific boulder up a particular hill. Implementation is starting to push that boulder up the right hill at the right time in the right way (fidelity). Institutionalization is getting the whole town to push the boulder to the top of the hill where it can stay year after year after year [20]. A description of the stages in detail and how it can be used to guide an institutional online/distance and on-campus learning implementation using a CMC portal is presented in the remainder of this section.

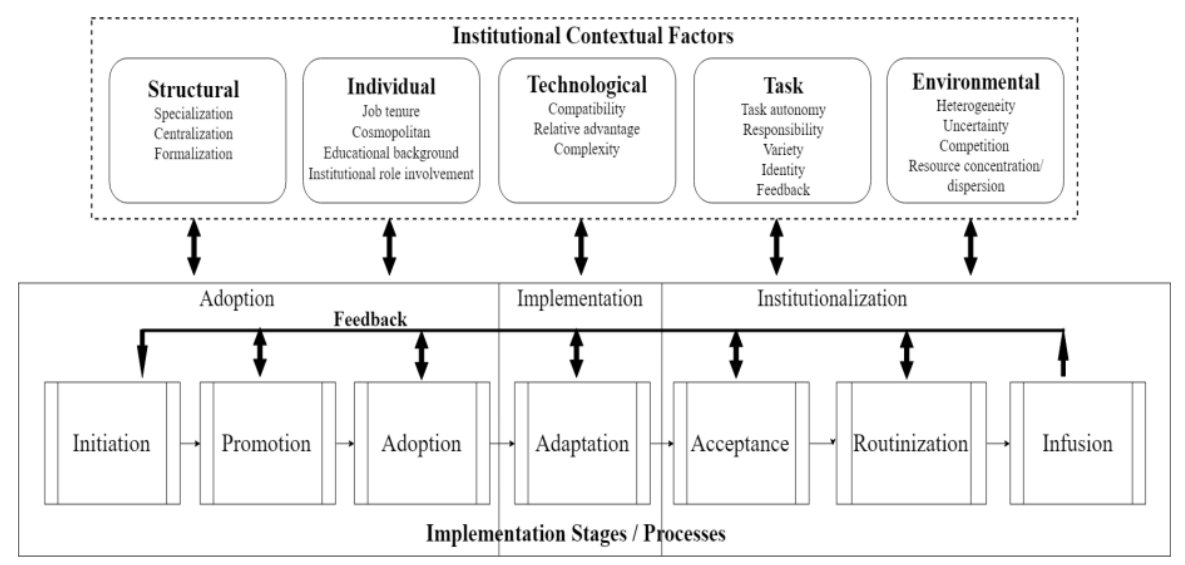

Fig. 2. Institutional CMC portal implementation framework 


\section{Discussion}

As demonstrated from the figure 2 above, the external boundary indicated by the dashed lines and labeled "Institutional Contextual Factors" at the upper part of the diagram shows the context within which the institutional implementation of the system occurs. The bidirectional arrows linking the external boundary to the main implementation process represents the contextual factors (structural, individual, technological, task, and environmental) that institutions need to be aware of in advance of, in the course of, and after the CMC portal implementation process.

At the lower part of the diagram is the actual implementation process which is divided into three (3) major phases(Adoption, Implementation and Institutionalization) and seven (7) sub-categories (initiation, promotion, adoption, adaptation, acceptance, routinization, and infusion) representing specific stages in the process and are linked by directional connectors that highlight the outputs from a particular stage. With the exception of initiation and infusion stages, the rest of the stages has a bidirectional arrow that connects into a solid black line showing a feedback process into the previous stages which highlight the possible consideration of activities that should have been considered in past stages yet were either not considered or the outputs from a specific stage was insufficient to facilitate the activities of the subsequent stage. It likewise shows an indication of how some of these activities can happen in parallel with other activities that are supposed to belong to a particular stage.

The solid black lines to which the arrows interface indicates the feedback process which is vital in each stage that enables corrective measures or improvements to be made to strengthen each stage outcome(s). Significantly, the arrow at the infusion stage which happens to be the last in the implementation process highlights the possible beginning of a new implementation objective, which can be an upgrade to a current objective or a reconsideration of activities that could have been properly addressed in the previous stage. The Successful implementation of a CMC portal in HEIs does not suggest an end to finding other ways by which the CMC portal could be utilized for but rather, it connotes the starting of a newer objective for the institution particularly where that objective was earlier not considered.

Processes preceding the physical deployment as well as processes after the deployment and utilization of the CMC portal is extensively considered as the entire implementation process. The conceptualization of these processes can bring about three broad stages within the entire process and it includes Adoption, Implementation, and institutionalization as seen in most educational change process literature. A discussion of the three broad stages of the framework in figure 2 is presented in the sections below.

\subsection{Adoption}

This stage takes a look at how the initiation, as well as the decision to introduce the CMC portal by the institutions, occur as far as who was behind and how the thought was consequently adopted or not. Institutional adoption of technological innovation requires a clear identification of a need or issue to be met and resolved by the introduction 
of the technology. In distinguishing the need or the issue, the role played by the institution through its mandated team is critical to ensuring that the identified solutions meet stakeholders' expectations. At the point when the technological introduction is enshrined in the institution's vision, mission and strategy, there can be a high achievement.

Institutional initiation: At this stage, Active as well as passive examining of institutional issues/opportunities and information technology solutions are birthed. Innovative ideas such as this, typically originate from either perceived institutional need (pull), perceived advantages of the technology (push) or both which leads to the identification of a match between CMC portal solutions and IT solutions in the institution.

Institutional planning: The introduction of a CMC portal system can only be successful when holistic planning takes into consideration available existing resources which include infrastructure, human, capital, and so is carried out.

Promotion: Promotion of the CMC portal system will enable stakeholders to know about the institutional aim for introducing the technology and enables them to become tied up with the process. Seminars and workshops organized by both internal as well as external facilitators can be a good way aimed at promoting and showing how the system works to stakeholders and these should even be possible sometime before the CMC portal system is introduced, in this manner enabling stakeholders to clear up their worries and misconceptions well before the system is ready and put into utilization.

Infrastructural readiness: The provision of internet/intranet, computer laboratories, computers, is important for any meaningful engagement with the CMC portal platform because the availability and readiness of the requisite IT infrastructure are critical to the successful implementation of the system. Reliable and accessible communication networks are needed by stakeholders both on-campus and off-campus to be able to engage with the CMC portal system. The system would have to be hosted on a dedicated computer server which may be internally done or externally outsourced weighing the advantages as well as disadvantages of both before a decision is made. Other issues concerning backup for the data as well as power is also of utmost great importance since the loss of data can happen if these are not taken care of.

Stakeholder involvement: Stakeholder involvement in the implementation of such innovation is critical to its success. Lecturers, students and some administrators who directly deal with students' issues can be described as the key stakeholders in the institutions. Other staff of the institutions who do not have a direct link with students as well as external people can be classified as secondary stakeholders. To achieve a successful implementation, it is critical to understand the role played by each of the stakeholders, their influence, as well as where and how they are involved in the implementation and utilization of the CMC portal.

Too often however this is inadequately addressed. These stakeholders must, therefore, be identified and their involvement strategically harnessed to ensure success as these are often not addressed inadequately.

Information technology staff readiness: The readiness of the ICT department/directorate is essential for a successful implementation. Most of these HEIs ICT staff 's original mandate has been to acquire, set up, manage as well as maintain all the IT software and hardware within the institution but with the introduction of applications for the running and management of institutions due to current developments in ICT, 
many technical competencies are now required from these ICT staffs. Especially, the ability to use different programming languages as well as databases which are essential in the setup and configuration of most of these CMC portal applications. The lack of these competencies by the ICT staff will warrant employing external assistance which will surely have financial implications on the institutions. The Moodle Open source application is lately being utilized by most HEIs and this application does require some kind of tune-up to suit the demands of the institutions.

External support availability: Any institution planning on having a successful implementation of such a system like the CMC portal will surely need the support of external person(s) or organizations if there is a lack of institutional capacity in terms of the setup and configuration needed for the smooth running of the system. the support from the external entities can come in the form of collaboration between institution who have successfully implemented such systems and have gained much experience as a result of that or through collaborations with organizations or individuals who have the expertise and can provide the needed support in such proprietary systems like the Moodle CMC portal. The achievement of the institutional goal concerning the implementation of such a system will be dependent on how they harness the support from these external entities and also, the determination of the stage(s) that the support will have a great impact is vital. The promotion, adaptation, as well as institutionalizing stages, can benefit greatly from this external support.

Consideration of core task (teaching and learning): Any educational institution's main core task is teaching and learning. The objectives for introducing an innovation like the CMC portal into the core task of the institution can be to support and facilitate students teaching and learning, broaden the scope of access to education and other policy initiatives by the government. Research has shown that a lot of students who enroll in courses offered online do quit without successful completion and others who enroll on the on/off-campus type using mediums like the CMC portal mostly becomes ineffective due to the lack of presence of the lecturers and so supporting and facilitating students teaching and learning through the CMC portal system needs a significant level of consideration as well as a careful design. If these considerations, as well as careful design, are not done, the likelihood of resistance from the stakeholders will be high due to conflict between the CMC portal software functionalities and traditional institutional practices and processes.

\subsection{Implementation (physical deployment)}

For any successful institutional CMC portal implementation, there must be a successful setup and configuration as well as a successful institutional process re-engineering. There are two different ways by which success can be achieved with the deployment of the system, the first is to adopt some of the Moodle's functionality to suit institutional requirements and the second is to adapt institutional processes to fit into the functionality of the Moodle CMC portal if there are solutions provided by the functions of the system to the challenges faced by the institution. At the point when institutions don't follow any of these two different ways, they are likely to face a rejection of the 
system which will lead to it not being integrated properly into institutional processes. The following are more clarifications concerning the implementation stage.

Installation and configuration: Getting software to perform its intended functions requires a hardware component since no software runs in isolation. Technical expertise is additionally required in other to set up, configure, and host the Moodle CMC portal software, however when these technical staff, as well as the resources needed for a successful implementation, isn't sufficient, there will be the need for external source to provide these two which will most likely add to the cost of implementation as well as bring about security and trust concerns. There can be the issue of trust regardless of whether the system setup and configuration is done in the institution. Numerous IT companies specializing in web hosting do offer services in Moodle application for institutions which include setting up, configuration and managing by which HEIs can settle on.

Courseware development and course site setup: The utilization of the CMC portal by stakeholders, especially the students will depend mostly on the level of courseware populated on the system since the primary role of any educational institution is to provide teaching and learning. Courseware involves audiovisuals, text, graphics and other materials deemed necessary by the institution, and so experts in these areas are needed in other to bring out materials that will be pedagogically accepted for online utilization. There are several ways by which the courseware can be developed and mounted and a decision to which way must be taken during inception by the institution to avoid undue delays or even not mounting of courses onto the portal. One way to do the development is to have it done in-house by each lecturer since they are the ones teaching the students, secondly, it can be done using a specially composed group within the institution, and lastly, it can be done using outsourced developers externally or buy from a commercial developer. The choice of which way to do the development will be dependent on whether the CMC portal is being used from the inception of the institution or used after the institution has been in existence for some time. This is because there will surely be courses being run traditionally in institutions which have been in existence before the portal introduction and so there will be some limitation as to the ways by which courseware can be developed.

The course site setup is also dependent on how the deployment is carried out, and this can be done by the trained lecturers or it can be done using a specialized group of people or department whom will assign roles to the lecturers to do modification if need be. A decision to which way the courseware development and setup must be taken and enforced before deployment.

Support staff involvement: To achieve the main purpose of any educational institution which is teaching and learning, various groups of staff work together to make it conceivable. The successful implementation of the CMC portal depends on the active involvement in the deployment processes of not only the lecturers and students but also the various departments like the admissions, examinations, library, students' accounts, and so on who are engaged with students' services. Neglecting such groups of staff will negatively affect the institutionalization of the system as in, the system will be seen as belonging to a particular group of people as opposed to being seen as an institutional one which leads to the resistance of the system. However, most institutions frequently 
don't consider these groups of people during these deployment stages, and to achieve a successful implementation, there should be the involvement of these groups of staff promptly within the deployment processes.

\subsection{Institutionalization}

The institutionalization phase which is the Post-implementation stage within the CMC portal system implementation process is where the system's functionalities are infused into the everyday activities carried out within the institution. At the institutionalization phase, the activities performed here can only be possible after a successful installation, systems configuration as well as a readily-accessible system for utilization. The evaluation of stakeholder acceptance of the systems' initial utilization is carried out at the phase, and then the necessary steps are taken to build up its acceptance if a negative acceptance is found during the initial assessment. Steps like the incorporation of the system into all stakeholders' daily activities as well as utilization of the full functionalities of the CMC portal system including integrating other IS used in the institution with the CMC portal system thereby having a bigger enterprise resource planning system are taken.

\section{Conclusion}

Holistic planning is needed before any of such systems are introduced in any institution and when such planning does not take place, the utilization of the system will be minimal when implemented which will be as a result of stakeholders resorting to the traditional methods of teaching, learning and the general administration of students.

To achieve a successful institutionalization of a CMC portal through the utilization of the proposed framework in figure 2, both the implementers and stakeholders must work together considering all institutional processes and activities as against the full functionalities of the CMC portal system. The main objectives for the introduction of a system like this can only be achieved when there is a successful institutionalization of the system within the institution.

Contextual factors will influence the proposed framework displayed in figure 2 above, and this will differ among various institutions. The majority of these factors will assume vital roles that will enable the institution to achieve its objectives for the introduction of the CMC portal. Some of the factors include management support and commitment as well as enforcement of utilization, institutional leadership, CMC portal development, stakeholders training, institutional processes to be supported known to developers, adequate technical staff, and availability of courseware.

Research into the implementation processes of a CMC portal in HEIs is just a fraction of how to achieve an effective CMC portal system. There is still the need for further research to be done on the process required for the integration of a CMC portal with other information systems forming an Enterprise resource planning system and also examine how institutional structures influence the level of stakeholders' utilization of a CMC portal system so as to contribute to a better institutionalization of CMC portals in 
HEIs. Since this research only focused on how the implementation of a CMC portal through the proposed framework is done in HEIs, future research can also address how the implementation can be carried out in the lower levels of education.

\section{References}

[1] R. E. Rice and D. Case, "Electronic Message Systems in the University: A Description of Use and Utility," Journal of Communication, 1983.

[2] E. Reid, "Electropolis: Communication and community on internet relay chat," 1991.

[3] E. Reid, "Electropolis: Communication and community on internet relay chat," 1991.

[4] R. Luppicini, "Review of computer-mediated communication research for education," Instructional Science, vol. 35, no. 2. pp. 141-185, 2007.

[5] W.-C. Lin, H. T. Huang, and H. Liou, "the Effects of Text-Based Scmc on Sla: a MetaAnalysis," Language Learning \& Technology, vol. 17, no. 2, pp. 123-142, 2013.

[6] W. H. DeLone and E. R. McLean, "The DeLone and McLean model of information systems success: A ten-year update," in Journal of Management Information Systems, 2003. https://doi.org/10.1080/07421222.2003.11045748

[7] R. Hirschheim, "Introduction to the special issue 'Quo Vadis TAM - Issues and reflections on technology acceptance research," Journal of the Association of Information Systems, 2007. https://doi.org/10.17705/1jais.00128

[8] D. Papachristos, N. Alafodimos, K. Arvanitis, M. Kalogiannakis, P. Kikilias, and S. Papadakis, "An Educational Model for Asynchronous E-Learning. A Case Study in a Higher Technology Education," International Journal of Advanced Corporate Learning (iJAC), vol. 3, no. 1, pp. 32-36, 2010. https://doi.org/10.3991/ijac.v3i1.987

[9] T. Browne, M. Jenkins, and R. Walker, "A longitudinal perspective regarding the use of VLEs by higher education institutions in the United Kingdom," Interactive Learning Environments, 2006. https://doi.org/10.1080/10494820600852795

[10] Papadakis, "Evaluating pre-service teachers' acceptance of mobile devices with regards to their age and gender: A case study in Greece," International Journal of Mobile Learning and Organisation, vol. 12, no. 4, pp. 336-352, 2018 . https://doi.org/10.1 504/ijmlo.2018.095130

[11] R. B. Cooper and R. W. Zmud, Cooper-1990-IT Implementation research- a technological diffusion approach.pdf. 1990.

[12] V. S. Lai, J. L. Guynes, and B. Bordoloi, "ISDN: Adoption and diffusion issues," Information Systems Management, 1993. https://doi.org/10.1080/10580539308906957

[13] G. Premkumar, K. Ramamurthy, and S. Nilakanta, "Implementation of electronic data interchange: An innovation diffusion perspective," Journal of Management Information Systems, 1994. https://doi.org/10.1080/07421222.1994.11518044

[14] I. Cho and Y. G. Kim, "Critical factors for assimilation of object-oriented programming languages," Journal of Management Information Systems, 2001.

[15] K. Lewin, "Group Decision and Social Change, in E. Newcombe and R. Harley, eds.," Readings in Social Psychology (New York: Henry Holt,), pp. 459-473, 1952.

[16] T. H. Kwon and R. W. Zmud, "Unifying the fragmented models of information systems implementation," in Critical issues in information systems research, 1987, pp. 227-251.

[17] B. L. Cooper, H. J. Watson, B. H. Wixom, and D. L. Goodhue, "Data warehousing supports corporate strategy at First American Corporation," MIS Quarterly: Management Information Systems, 2000. https://doi.org/10.2307/3250947 
[18] J. Peppard and J. Ward, "Beyond strategic information systems: Towards an IS capability," Journal of Strategic Information Systems, 2004. https://doi.org/10.1016/j.jsis.2004.02.002

[19] A. Beaudry and A. Pinsonneault, "Advancing the Theory of Infusion: An Appropriation Model of the Infusion Process," Cahier du GreSI, no. 99, 1999.

[20] B. Kwofie, "E-learning implementation in higher education institutions," Aalborg University, 2015.

[21] C. R. Graham, W. Woodfield, and J. B. Harrison, "A framework for institutional adoption and implementation of blended learning in higher education," Internet and Higher Education, 2013. https://doi.org/10.1016/j.iheduc.2012.09.003

\section{$7 \quad$ Authors}

Emmanuel Dortey Tetteh is a Ph.D. Candidate of School of Information and Software Engineering from University of Electronic Science and Technology of China, Chengdu, China.

Zhiguang Qin was a former Dean of the School of Information and Software Engineering at the University of Electronic Science and Technology of China (UESTC), where he was also Director of the Key Laboratory of New Computer Application Technology and Director of UESTC-IBM Technology Center.

Benjamin Kwofie is a lecturer at the Koforidua Technical University in Koforidua, Ghana. He is at the computer science department of the faculty of applied science and technology.

Article submitted 2019-09-08. Resubmitted 2019-11-08. Final acceptance 2019-11-08. Final version published as submitted by the authors. 